\title{
Asma brônquica descompensada no atendimento de emergência em um município de Pernambuco durante a pandemia da COVID-19: Relato de caso
}

\author{
Acute bronchial asthma in emergency care in a city of Pernambuco during the COVID-19 \\ pandemic: Case report
}

Asma bronquial descompensada en atención de emergencia en una ciudad de Pernambuco durante

la pandemia COVID-19: Reporte de caso

Thyago de Oliveira Afonso ORCID: https://orcid.org/0000-0001-7616-9011 Universidade Federal de Pernambuco, Brasil

E-mail: thyago.oafonso@gmail.com

Samuel Lopes dos Santos ORCID: https://orcid.org/0000-0003-3375-9171 Universidade Federal do Piauí, Brasil

E-mail: samuellopes121314@gmail.com

Gustavo Baroni Araújo

ORCID: https://orcid.org/0000-0002-3162-7477

Universidade Estadual de Londrina, Brasil

E-mail: gustavobaroni13@hotmail.com

Igor de Oliveira Carvalho

ORCID: https://orcid.org/0000-0002-7487-8073

Universidade Federal de Goiás, Brasil

E-mail: igorolivcarvalho@gmail.com

Guilherme Dantas Borges

ORCID: https://orcid.org/0000-0002-3415-8725

Universidade de Vassouras, Brasil

E-mail: gdantasborges@gmail.com

Caio Victor Muniz de Lima Barros

ORCID: https://orcid.org/0000-0001-8052-2543

Centro Universitário UniFacid Wyden, Brasil

E-mail: caiovmlbb@gmail.com

Simone Barroso de Carvalho

ORCID: https://orcid.org/0000-0001-7428-8420

Universidade Federal do Piauí, Brasil

E-mail: simonebcarvalho2009@hotmail.com

Elvira Marques da Luz Dias

ORCID: https://orcid.gov/0000-0001-7966-0877 Universidade Brasil, Brasil

E-mail: elviraluzz@hotmail.com

Marília Girão de Oliveira Machado

ORCID: https://orcid.gov/0000-0001-7163-9061

Universidade de Fortaleza, Brasil

E-mail: mariliagirao05@hotmail.com

Rosanna Rafena Ribeiro Barbosa

ORCID: https://orcid.org/0000-0003-1777-2245

Associação de Ensino Superior do Piauí, Brasil E-mail: rrafena@live.com

Ana Izabel Aparecida Vieira

ORCID: https://orcid.org/0000-0001-6617-7499

Universidade de Vassouras, Brasil

E-mail: anaizabelavieira@gmail.com

Luziane Cardoso Costa

ORCID: https://orcid.org/0000-0003-4579-8223

Escola Superior da Amazônia, Brasil

E-mail: luzianeccardoso@gmail.com

Leandro Luiz da Silva Loures

ORCID: https://orcid.org/0000-0001-8863-8858

Universidade Federal de Juiz de Fora, Brasil

E-mail: leandrolouresnutri@gmail.com 


\author{
Bernardo da Luz Barbosa \\ ORCID: https://orcid.org/0000-0002-3866-4887 \\ Universidade de Vassouras, Brasil \\ E-mail: bernardolbarbosa@hotmail.com \\ Gustavo Martins de Araujo Porto \\ ORCID: https://orcid.org/0000-0003-2930-3534 \\ Universidade de Vassouras, Brasil \\ E-mail: gustavoportoaraujo@gmail.com \\ Isadora Nunes Delaia \\ ORCID: https://orcid.org/0000-0002-0336-848X \\ Universidade de Vassouras, Brasil \\ E-mail: isanunes.del@gmail.com \\ Erica Patrícia Dias de Sousa \\ ORCID: https://orcid.org/0000-0001-7675-902X \\ UNIPOS, Brasil \\ E-mail: ericaigsousa@gmail.com \\ Mariana Teixeira da Silva \\ ORCID: https://orcid.org/0000-0002-1904-0921 \\ Universidade Federal do Piauí, Brasil \\ E-mail: marytsilva44@gmail.com \\ Camila Leanne Teixeira Coelho de Sousa \\ ORCID: https://orcid.org/0000-0002-2525-0335 \\ Universidade Federal do Maranhão, Brasil \\ E-mail: camilaleanne@ hotmail.com \\ Ana de Cássia Ivo Dos Santos \\ ORCID: https://orcid.org/0000-0001-5549-1263 \\ Universidade Federal do Piauí, Brasil \\ E-mail: a.cassia.enfermagem@gmail.com \\ Francisca Agda Oliveira Dias \\ ORCID: https://orcid.org/0000-0002-1618-5180 \\ Instituto Dom Bosco, Brasil \\ E-mail:agdadiascx@gmail.com
}

\begin{abstract}
Resumo
A asma brônquica é uma doença crônica das vias aéreas, heterogênea e etiologia desconhecida. No Brasil, a asma brônquica descompensada é a quarta maior causa de internações, com maior incidência nas regiões Nordeste e Sudeste, apresentando índices de mortalidade descendentes. Os sintomas e sinais mais comuns são dispneia, sibilos, opressão torácica retroesternal e tosse, decorrentes do processo inflamatório, com hiperreatividade brônquica e broncoconstrição. O presente artigo objetiva alertar os profissionais de saúde sobre o diagnóstico precoce e tratamento da asma exacerbada em emergência no contexto pandêmico da COVID-19, através deste relato de caso. Tratando também do diagnóstico diferencial em sintomáticos respiratórios para COVID-19 com avaliação de riscos epidemiológicos e radiografia, pouco sensíveis e específicos para COVID-19, mas amplamente disponíveis. Este relato de caso trata-se de uma mulher de 19 anos, caucasiana, solteira, natural e procedente de Caruaru-PE, com queixas de desconforto torácico de baixa intensidade, dispneia e fadiga há três dias, apresentando exacerbação dos sintomas nas últimas quatro horas, com diagnóstico prévio de asma desde a infância, atualmente com tratamento interrompido por medo de agravamento para a COVID-19 e despertar noturno todos os dias durante o último mês. Após conduta terapêutica e melhora clínica, a paciente obteve alta com orientações sobre continuidade do corticoide oral, retorno à terapia de controle da asma brônquica, necessidade do acompanhamento na Atenção Primária à Saúde (APS) e sintomas de gravidade para a asma brônquica e COVID-19 que indicam a necessidade de atendimento emergencial.
\end{abstract}

Palavras-chave: Asma; Exacerbação de sintomas; Terapêutica; Atendimento de emergência; Infecções por coronavírus.

\begin{abstract}
Bronchial asthma is a chronic disease of the airways, heterogeneous and of unknown etiology. In Brazil, decompensated bronchial asthma is the fourth leading cause of hospitalizations, with a higher incidence in the Northeast and Southeast regions, with descending mortality rates. The most common symptoms and signs are dyspnea, wheezing, retrosternal chest tightness and cough, resulting from the inflammatory process, with bronchial hyperreactivity and bronchoconstriction. This article aims to alert health professionals about the early diagnosis and treatment of exacerbated asthma in an emergency in the pandemic context of COVID-19, through this case report. Also dealing with the differential diagnosis in respiratory symptoms for COVID-19 with assessment of epidemiological risks and radiography, not very sensitive and specific for COVID-19, but widely available. This case report is about a 19-year-old Caucasian woman, single, born in Caruaru-PE, with complaints of low intensity chest discomfort, dyspnea and fatigue for three days, presenting exacerbation of symptoms in the last four hours, with previous diagnosis of asthma since childhood, currently with treatment interrupted due to fear of worsening for
\end{abstract}


COVID-19 and waking up at night every day during the last month. After therapeutic conduct and clinical improvement, the patient was discharged with instructions on continuity of oral corticosteroids, return to bronchial asthma control therapy, need for follow-up in Primary Health Care (PHC) and symptoms of severity for bronchial asthma and COVID- 19 that indicate the need for emergency care.

Keywords: Asthma; Symptom flare up; Therapeutics; Ambulatory care; Coronavirus infections.

\section{Resumen}

El asma bronquial es una enfermedad crónica de las vías respiratorias, heterogénea y de etiología desconocida. En Brasil, el asma bronquial descompensada es la cuarta causa principal de hospitalizaciones, con mayor incidencia en las regiones Nordeste y Sudeste, con tasas de mortalidad decrecientes. Los signos y síntomas más frecuentes son disnea, sibilancias, opresión torácica retroesternal y tos, consecuencia del proceso inflamatorio, con hiperreactividad bronquial y broncoconstricción. Este artículo tiene como objetivo alertar a los profesionales de la salud sobre el diagnóstico y tratamiento precoz del asma exacerbada en una emergencia en el contexto pandémico de COVID-19, a través de este caso clínico. También trata el diagnóstico diferencial en síntomas respiratorios de COVID-19 con evaluación de riesgo epidemiológico y radiografía, que es insensible y específico para COVID-19, pero ampliamente disponible. El presente caso clínico trata de una mujer caucásica de 19 años, soltera, nacida en Caruaru-PE, que consulta por malestar torácico de baja intensidad, disnea y cansancio durante tres días, presentando agudización de los síntomas en las últimas cuatro horas, con diagnóstico previo por asma desde la niñez, actualmente con tratamiento interrumpido por temor a empeorar por COVID-19 y despertarse por la noche todos los días durante el último mes. Tras la conducta terapéutica y la mejoría clínica, el paciente fue dado de alta con instrucciones de continuidad de corticoides orales, retorno a terapia de control del asma bronquial, necesidad de seguimiento en Atención Primaria de Salud (APS) y síntomas de gravedad por asma bronquial y COVID-19 que indicar la necesidad de atención de emergencia.

Palabras clave: Asma; Exacerbación de los síntomas; Terapia; Cuidados de emergencia; Infecciones por coronavirus.

\section{Introdução}

A asma é uma doença crônica das vias aéreas, prioritariamente das inferiores, heterogênea, com etiologia desconhecida, e com presença de várias células e elementos imunológicos em sua patogenia. Definida pela história de sintomas respiratórios, como sibilos, dispneia, opressão torácica retroesternal e tosse, os quais variam com o tempo e intensidade, associados a fatores de gatilho, como exercício, alérgicos e fatores irritativos, e à limitação de fluxo aéreo (Boulet et al., 2019; Holgate et al., 2015; Papi et al., 2018). Tais episódios são explicados pela evolução da inflamação crônica e hiper-reatividade brônquica, com episódios recorrentes de obstrução generalizada das vias aéreas, com reversibilidade espontânea ou com tratamento (Holgate et al., 2015; Longo et al., 2020; Papi et al., 2018).

A prevalência mundial varia entre $4,7 \%$ a 30,5\%, com média 20\%, acometendo cerca de 300 milhões de pessoas, afetando indivíduos de todas as idades, etnias e sexo, embora apresente maior incidência em pessoas até 25 anos do sexo masculino (Boulet et al., 2019). No contexto brasileiro, o país apresenta-se como oitavo país na incidência de asma no mundo, com 20 milhões de indivíduos (Brasil, 2020; Boulet et al., 2019).

A mortalidade desta doença está diretamente relacionada com o manejo adequado dos quadros sintomatológicos e acesso aos serviços de saúde no mundo (Lundback et al., 2016). No país, a mortalidade por asma é descendente, explicada pelo maior acesso da população ao Sistema Único de Saúde (SUS) e uso de corticoides (Cardoso et al., 2016), no entanto, ainda é vista como a quarta maior causa de internamento em 2020, principalmente nas regiões Nordeste e Sudeste, com 2339 mortes por ano relatadas em todo país (Brasil, 2021).

O diagnóstico é dado pelo histórico ou presença de sintomas associados à limitação variável do fluxo respiratório, o qual pode ser avaliado através de espirometria com ou sem broncodilatador, hiperresponsividade das vias aéreas e outros testes/escores de avaliação, como Escore Castro modificado por Guilbert (Pizzichini et al., 2020). Ademais, outros diagnósticos diferenciais devem ser realizados, principalmente em grupos específicos, de gravidade e frente a quadros epidemiológicos instaurados localmente, como para a doença do coronavírus (COVID-19) (Li et al., 2020; Liu e tal., 2020; WHO, 2020; Zou et al., 2020).

A solicitação de exames complementares e tratamento devem ser direcionados para a história pregressa, apresentação 
de sintomas agudos e de gravidade, comorbidades, medicações em uso, avaliação de riscos modificáveis e satisfação do paciente. Desta forma, para o controle da asma é objetivado a reavaliação periódica do paciente e suas metas, através do ajuste de doses medicamentosas, estratégias não-medicamentosas e reavaliação de resposta (Boulet et al. 2019; Pizzichini et al., 2020). Sendo assim, o presente artigo tem como objetivo alertar os profissionais de saúde sobre o diagnóstico precoce e tratamento da asma exacerbada em emergência no contexto pandêmico da COVID-19, através deste relato de caso.

\section{Metodologia}

Trata-se de um estudo observacional e descritivo com relato de caso com informações obtidas em uma unidade de pronto atendimento por meio de consulta médica, entrevista com o paciente, revisão de exames complementares e outros métodos diagnósticos que o paciente foi submetido e revisão de literatura. A identidade do paciente foi preservada e os exames complementares, como o de imagem, não registrados neste artigo para assegurar o anonimato do paciente.

\section{Relato de Caso}

Paciente feminina com 19 anos, caucasiana, solteira, natural e procedente de Caruaru, Pernambuco, Brasil, não etilista e não tabagista, admitida em Unidade de Pronto Atendimento (UPA) - 24h do bairro Vassoural em 14 de março de 2021, queixando-se de desconforto torácico de baixa intensidade, dispneia e fadiga há três dias, principalmente à noite, apresentando exacerbação dos sintomas nas últimas quatro horas. Relata diagnóstico prévio de asma desde a infância, atualmente com tratamento interrompido por medo de agravamento para a COVID-19. Informa que há um mês tem despertado à noite, pelo menos uma vez todos os dias, sem realizar terapia de resgate para exacerbação da asma brônquica. Quando questionada sobre medicações em uso, informa o uso prévio de budesonida 100 micrograma por spray (100mcg/spray) em duas doses inalatórias de 12/12 horas ao dia e fumarato de formoterol de seis micrograma por spray ( $6 \mathrm{mcg} / \mathrm{spray}$ ) em dose inalatória de 12/12 horas ao dia. Desconhece contato prévio com pacientes diagnosticados com COVID-19, mas afirma sintomas respiratórios em seus pais, que residem na mesma casa. Nega diabetes mellitus (DM), hipertensão arterial sistêmica (HAS), alergias prévias, atividades físicas regulares e acompanhamento nutricional.

Ao exame físico ectoscópico geral, mostrava-se em estado geral regular (EGR), normocorada, anictérica, acianótica, afebril, hidratada, taquipneica (32 incursões respiratórias por minuto - irpm), taquicárdica (105 batimentos por minuto - bpm),

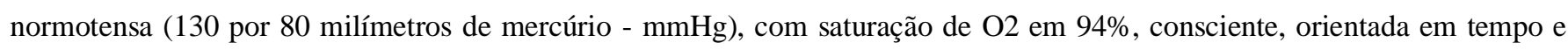
espaço, ativa e colaborativa. Ao aparelho cardiovascular e respiratório, apresentava bulhas normofonéticas (BNF) em dois tempos (2T), sem sopros (s/p), com pulsos simétricos, cheios e rítmicos, murmúrios vesiculares presentes (MV+) com presença de sibilos em ambos hemitórax (AHT), com expansividade torácica preservada, sem uso de musculatura acessória e sem batimento de asa de nariz. Ao exame físico dos demais aparelhos, não foram encontradas anormalidades significativas.

Após queixa principal, história da doença atual e exames físicos da paciente, foram instituídas hipóteses diagnósticas de asma brônquica descompensada e interrogada COVID-19. Realizada radiografia de tórax com incidências em perfil e póstero-anterior (PA) sem anormalidades ou achados indicativos de infecção. O exame de hemograma completo apresentavase sem alterações significativas.

A conduta instituída nas duas primeiras horas foi iniciar sulfato de salbutamol (Aerolin®) de $100 \mathrm{mcg} / \mathrm{spray}$ em quatro jatos inalados a cada $20 \mathrm{~min}$. A paciente também foi submetida à corticoterapia sistêmica, através de dois comprimidos de prednisona de 20 miligramas por comprimido (mg/cp) por via oral.

$\mathrm{Na}$ reavaliação, apresentava melhores condições clínicas e foi dada alta com orientação para manter corticoide sistêmico com prednisona (20mg/cp) via oral de $24 / 24 \mathrm{~h}$ durante cinco dias, retornar a terapia de controle da asma brônquica 
com budesonida e fumarato de formoterol em mesmas doses anteriormente prescritas, assim como para as doses de resgate, reforçada a necessidade de acompanhamento da paciente na Atenção Primária à Saúde (APS). Para tranquilizar a paciente sobre seus medos foi orientado sobre demais sintomas de gravidade para a asma brônquica e COVID-19 que indicam a necessidade de atendimento médico de urgência.

\section{Discussão}

Segundo o Global Initiative for Asthma, a asma brônquica apresenta-se como uma doença heterogênea, geralmente caracterizada por inflamação crônica das vias aéreas e remodelação variável, sendo a mais comum dos pulmões. Definida pela história de sintomas respiratórios, tais como sibilos, dispneia, opressão torácica retroesternal e tosse, variando conforme tempo e intensidade, com associação à limitação variável do fluxo aéreo, causando sérios sintomas em pacientes asmáticos e elevando o custo para os sistemas de saúde mundiais (Boulet et al., 2020). A paciente em questão apresentou-se com sibilos, dispneia e opressão torácica, que mesmo com saturação de oxigênio satisfatória, havia causado debilidade respiratória e fadiga.

A asma é um distúrbio extremamente comum mundialmente, acometendo cerca de 300 milhões de pessoas, com prevalência variando entre $4,7 \%$ a 30,5\%, com média de $20 \%$, tendo o início dos sintomas antes dos 25 anos de idade, mesmo acometendo qualquer idade e sexo (Boulet et al., 2020; Holgate et al., 2015; Papi et al., 2018). O Brasil ocupa a oitava posição em prevalência de asma, com aproximadamente 20 milhões de pessoas, com maior representação nas regiões Sul e Sudeste em 2020 (Brasil, 2021). Porém, quando analisados os números de internações segundo região no país ainda é observado que no ano de 2020 houveram 47.731 internações por asma, representando a quarta maior causa de internamento no Brasil, com maior número de internações nas regiões Nordeste com 17.570 internações (36,81\%) e Sudeste com 15.504 (32,48\%), seguido das regiões Sul com 6.557 internações (13,73\%), Norte com 4.427 internações (9,27\%) e Centro-oeste com 3.673 (7,69\%) (Brasil, 2021; Cardoso et al., 2017).

Mesmo considerando a asma como importante causa de internamentos e custos para o serviço de saúde, há representativa queda nos números de novos casos e agravamento da doença, explicados pelo acesso aos serviços de saúde, manejo correto e inserção de corticoides inalatórios na terapia de controle e resgate. Mesmo com tais medidas, o Brasil registra anualmente uma mortalidade de aproximadamente 2.339 mortes por asma descompensada que pode ser ocasionada por fatores genéticos, ambientais, má adesão ao tratamento e dificuldades de acesso a serviços de saúde (Cardoso et al., 2017).

A origem e gravidade da asma são impulsionados pela presença de fatores genéticos e ambientais, apresentando sintomas mais comumente durante a infância dada a associação de sensibilização dependente de IgE e outros alérgenos ambientais. No entanto, a asma pode ter início também em idade adulta, com correlação direta à intolerância medicamentosa, obesidade prévia e neutrofilia, na asma neutrofílica (Holgate et al., 2015; Papi et al., 2018). A progressão da doença envolve um desenvolvimento histórico, no qual está fortemente relacionado à sensibilização alérgica e declínio da função pulmonar ao longo do tempo (Longo et al., 2020).

No ponto de vista imunológico, a asma é dividida em reação imediata e tardia, relacionada a estímulos eosinofílicos, linfocítico e seus mediadores (gamainterferona e interleucinas), através das vias dos linfócitos T helper 2 (Th2) e não Th2. Devido à reação imunopatogênica, os sinais e sintomas de apresentação na asma é resultado da diminuição do calibre das vias aéreas, dada hipersecreção de muco e edema, hipersensibilidade a estímulos pela destruição de células epiteliais das vias aéreas pela inflamação crônica e posterior exposição de terminações nervosas aos estímulos externos e internos. Tais estímulos são fatores desencadeantes, como poeira, poluição, bactérias, vírus, medicamentos, obesidade, fumo, prática de exercícios, mudança de temperatura, exposições ocupacionais e até não ter fatores desencadeantes identificáveis, os quais podem exacerbar os sintomas da asma e causar agravamento no fluxo de ar respiratório pela broncoconstrição (Holgate et al., 2015; Papi et al., 2018). 
Desta maneira, a avaliação inicial do paciente com asma descompensada deverá ser direcionada para compreender principalmente a sintomatologia apresentada, frequência, fatores de melhora e piora, uso medicamentoso, adesão do tratamento (quando já iniciado), fatores desencadeantes presentes e comorbidades. Ao exame físico, as características mais comuns são taquipneia, taquicardia e pulso paradoxal (Boulet et al., 2019; Pizzichini et al., 2020), com os dois primeiros apresentados pela paciente do caso. Normalmente, a saturação de oxigênio encontra-se próxima dos $90 \%$, divergindo do obtido na paciente $(94 \%)$.

Ao exame físico do tórax é observado uso de musculatura acessória, hiperinsuflação torácica, fase expiratória prolongada, hipertimpanismo à percussão, frêmito tóraco-vocal diminuído e presença de sibilos difusos e polifônicos à ausculta. A ausência de sibilos pode indicar sinal de gravidade e obstrução grave das vias aéreas. Os achados relacionados a crises graves incluem ansiedade, sudorese, tiragem intercostal ou supraesternal, inquietação, pulso paradoxal, alteração no nível de consciência e "tórax silencioso" (Pizzichini et al., 2020).

O diagnóstico é dado através de anamnese e exame físico, com histórico ou presença de sinais respiratórios consistentes com asma associado a limitação variável do fluxo de ar expiratório, preferencialmente deve ser realizado antes de iniciar terapia medicamentosa, tornando o diagnóstico mais complicado após tratamento estabelecido. A limitação do fluxo de ar expiratório pode ser registrada através de exames complementares, como espirometria, medida de pico de fluxo expiratório, verificação de hiperresponsividade das vias aéreas e critérios/score, como Escore Castro modificado por Guilbert (Boulet et al., 2019; Pizzichini et al., 2020).

Com relação aos diagnósticos diferenciais e solicitação de exames complementares, estes devem ser direcionados pela história da doença e clínica apresentada do paciente, e quadros epidemiológicos presentes de surto, endemia, epidemia ou pandemia, solicitando-se radiografia de tórax, gasometria arterial, hemograma, eletrólitos, eletrocardiograma e peak flow (Pizzichini et al., 2020).

No contexto atual da pandemia de COVID-19, ocasionada pelo novo coronavírus da síndrome respiratória aguda grave 2 (SARS-COV-2), qualquer sintoma respiratório em pacientes atendidos em emergência pode ser característico de infecção pelo vírus, principalmente quando relatado contato prévio com pessoas diagnosticadas (Liu et al., 2020; Mentes et al., 2021). Considerado o padrão ouro para diagnóstico da COVID-19, o teste de reação da transcriptase reversa seguida pela reação em cadeia da polimerase (RT-PCR) é dado pela da presença do ácido ribonucleico (RNA) viral na amostra de nasofaringe. Além deste, há outros exames e testes que auxiliam no diagnóstico da COVID-19, como os exames de sorologia de imunoglobulina (Ig) M (IgM) e IgG, e testes imunocromatográficos rápidos IgM/IgG, estes possuindo menor especificidade e sensibilidade quando comparado ao RT-PCR (Brasil, 2020; Hartmann-Boyce et al., 2020; Mendes et al., 2021).

Dentro de uma unidade de emergência, o paciente sintomático respiratório deverá ser submetido aos testes diagnósticos para COVID-19 disponíveis, segundo recomendação do Ministério da Saúde (Brasil, 2020). Contudo, a disponibilidade desses testes é falha em diversos locais, tornando o diagnóstico específico mais complicado. Nesse ponto, torna-se um desafio o diagnóstico diferencial entre a asma e COVID-19, podendo o médico de plantão utilizar-se de exames de imagem, contextos de exposição ao risco e epidemiológicos para estratificar o risco e possível diagnóstico da COVID-19 (Liu et al., 2020; Mendes et al., 2021).

Os fatores de risco para agravamento da COVID-19 estão diretamente relacionados aos processos inflamatórios prévios, como obesidade, HAS e DM descompensada, quadros de imunossupressão e asma descompensada (Li et al., 2020; Zhou et al., 2020). Assim, a asma descompensada torna-se fator de risco para exacerbações e agravamento da COVID-19, e diante disto é necessário o controle e classificação da doença (crise leve a moderada ou crise grave) para individualização terapêutica e avaliação de terapias de suporte, como ventilação mecânica não invasiva ou invasiva e internamento em unidade de terapia intensiva (Pizzichini et al., 2020). 
O controle e classificação deve seguir instrumentos qualificados pelo GINA, Asthma Control Questionnaire com 7 itens (ACQ-7) ou Asthma Control Test (ACT), recomendados pela Sociedade Brasileira de Pneumologia e Tisiologia. Esses testes qualificam os pacientes em passos que serão essenciais para manejo terapêutico com corticoide inalatório, corticoide sistêmico, anti-IgE, beta-2-agonista de longa duração, beta-2-agonista de curta duração, oxigenoterapia ou ipratrópio (Pizzichini et al., 2020).

Diante do contexto COVID-19, não é recomendada a terapia com nebulização com beta-2-agonista de curta duração ou outras terapias que gerem aerossóis e possam contaminar o ambiente com partículas do SARS-COV-2, como avaliado para a paciente do caso (Mendes et al., 2021; Pizzichini et al., 2020).

Ademais, o tratamento e prognóstico após alta devem objetivar: ajuste terapêutico, quando necessário e confirmado o diagnóstico; controlar sintomas, fatores de risco e comorbidades; educar quanto às técnicas de inalação, medicamentos, estratégias não-farmacológicas e metas para o paciente; rever as respostas do ajuste nos sintomas, exacerbações, efeitos colaterais e função pulmonar; orientar sobre seguimento e acompanhamento na Atenção Primária à Saúde (Gupta et al, 2019; Wu et al., 2019), conforme orientado a paciente deste relato.

\section{Conclusão}

O presente artigo objetivou alertar os profissionais de saúde sobre o diagnóstico precoce e tratamento da asma exacerbada em emergência no contexto pandêmico da COVID-19. Tratando também do diagnóstico diferencial em sintomáticos respiratórios para COVID-19 com avaliação de riscos epidemiológicos e exames de imagem, pouco sensíveis e específicos para COVID-19, mas amplamente disponíveis e sugerindo um diagnóstico provável que deve ser avaliado com a clínica. No mais, reforça-se a importância da reavaliação, ajuste, satisfação do paciente, controle de sintomas e fatores de risco durante a terapêutica para asma, e orientação de acompanhamento na Atenção Primária à Saúde.

\section{Referências}

Boulet, L. P. et al. (2019). The global initiative for asthma (GINA): 25 years later. European Respiratory Journal, 54(2).

Brasil. Ministério da Saúde. (2021). Sistema de Informações Hospitalares do SUS (SIH/SUS). http://www2.datasus.gov.br/DATASUS/in dex.php?area=060502.

Brasil. Ministério da Saúde. (2020). Orientações para manejo de pacientes com COVID-19. https://portalarquivos.saude.gov.br/images/pdf/2020/J une/18/Covid19-Orientac--o--esManejoPacientes.pdf.

Cardoso, T. D. A., Roncada, C., Silva, E. R. D., Pinto, L. A., Jones, M. H., Stein, R. T., \& Pitrez, P. M. (2017). The impact of asthma in Brazil: a longitudinal analysis of data from a Brazilian national database system. Jornal brasileiro de pneumologia, 43(3), 163-168.

Chesné, J. et al. (2014). IL-17 in severe asthma. Where do we stand? Am. J. Respir. Crit. Care Med. 190, 1094-1101.

Custovic, A. (2015) To what extent is allergen exposure a risk factor for the development of allergic disease? Clin. Exp. Allergy 45, 54-62

Gupta, S., Price, C., Agarwal, G., Chan, D., Goel, S., Boulet, L. P., \& Straus, S. E. (2019). The Electronic Asthma Management System (eAMS) improves primary care asthma management. European Respiratory Journal, 53(4).

Harkness, L. M., Ashton, A. W. \& Burgess, J. K. (2015). Asthma is not only an airway disease, but also a vascular disease. Pharmacol. Ther. 148, 17-33 (2015).

Hartmann-Boyce, J., Gunnell, J., Drake, J., Otunla, A., Suklan, J., Schofield, E., \& Dennison, P. (2020). Asthma and COVID-19: review of evidence on risks and management considerations. BMJ Evidence-Based Medicine.

Holgate, S., Wenzel, S., Postma, D. et al. (2015). Asthma. Nat Rev Dis Primers 1, 15025. https://doi.org/10.1038/nrdp.2015.25

Li, X., Xu, S., Yu, M., Wang, K., Tao, Y., Zhou, Y., \& Zhao, J. (2020). Risk factors for severity and mortality in adult COVID-19 inpatients in Wuhan. Journal of Allergy and Clinical Immunology, 146(1), 110-118.

Liu, S., Zhi, Y., \& Ying, S. (2020). COVID-19 and asthma: reflection during the pandemic. Clinical reviews in allergy \& immunology, 59, 78-88.

Longo, D. L., Kasper, D. L., Jameson, J. L., Fauci, A. S., Hauser, S. L., \& Loscalzo, J. (2020). Medicina interna de Harrison. In Medicina interna de Harrison. 20. ed. Porto Alegre. Artmed. 
Research, Society and Development, v. 10, n. 5, e52910514959, 2021 (CC BY 4.0) | ISSN 2525-3409 | DOI: http://dx.doi.org/10.33448/rsd-v10i5.14959

Lundbäck, B., Backman, H., Lötvall, J., \& Rönmark, E. (2016). Is asthma prevalence still increasing?. Expert review of respiratory medicine, 10(1), 39-51.

Martinez-Gonzalez, I., Steer, C. A. \& Takei, F. (2015). Lung ILC2s link innate and adaptive responses in allergic inflammation. Trends Immunol. 36, 189-195 (2015).

Mendes, N. F., Jara, C. P., Mansour, E., Araújo, E. P., \& Velloso, L. A. (2021). Asthma and COVID-19: a systematic review. Allergy, Asthma \& Clinical Immunology, 17(1), 1-12.

Pizzichini, M. M. M., Carvalho-Pinto, R. M. D., Cançado, J. E. D., Rubin, A. S., Cerci Neto, A., Cardoso, A. P., \& Cukier, A. (2020). Recomendações para o manejo da asma da Sociedade Brasileira de Pneumologia e Tisiologia-2020. Jornal Brasileiro de Pneumologia, 46(1).

Papi, A. et al. (2018). Asthma, Asthma-Science Direct, 391(24), 783-800.

WHO. World Health Organization. (2020). Clinical management of severe acute respiratory infection when novel coronavirus ( nCoV) infection is suspected: interim guidance, 25 January 2020 (No. WHO/nCoV/Clinical/2020.2). World Health Organization.

Wu, T. D., Brigham, E. P., \& McCormack, M. C. (2019). Asthma in the primary care setting. Medical Clinics, $103(3), 435-452$.

Zhou, F., Yu, T., Du, R., Fan, G., Liu, Y., Liu, Z., \& Cao, B. (2020). Clinical course and risk factors for mortality of adult inpatients with COVID-19 in Wuhan, China: a retrospective cohort study. The lancet, 395(10229), 1054-1062. 